\title{
Two-time height distribution for 1D KPZ growth: the recent exact result and its tail via replica
}

\author{
Jacopo de Nardis ${ }^{1}$ and Pierre Le Doussal ${ }^{2}$ \\ ${ }^{1}$ Département de Physique, Ecole Normale Supérieure, PSL Research University, \\ CNRS, 24 rue Lhomond, 75005 Paris, France \\ 2 CNRS-Laboratoire de Physique Théorique de l'Ecole Normale Supérieure, 24 rue \\ Lhomond, 75231 Paris Cedex, France \\ E-mail: jacopo.de.nardis@phys.ens.fr \\ E-mail: ledou@lpt.ens.fr
}

\begin{abstract}
We consider the fluctuations in the stochastic growth of a one-dimensional interface of height $h(x, t)$ described by the Kardar-Parisi-Zhang (KPZ) universality class. We study the joint probability distribution function (JPDF) of the interface heights at two-times $t_{1}$ and $t_{2}>t_{1}$, with droplet initial conditions at $t=0$. In the limit of large times this JPDF is expected to become a universal function of the time ratio $t_{2} / t_{1}$, and of the (properly scaled) heights $h\left(x, t_{1}\right)$ and $h\left(x, t_{2}\right)$. Using the replica Bethe ansatz method for the KPZ equation, in [1] we obtained a formula for the JPDF in the (partial) tail regime where $h\left(x, t_{1}\right)$ is large and positive, subsequently found in excellent agreement with experimental and numerical data [2]. Here we show that our results are in perfect agreement with Johansson's recent rigorous expression of the full JPDF [3], thereby confirming the validity of our methods.
\end{abstract}

\section{Introduction}

Growth processes are natural phenomena occurring when a stable phase of a generic system expands into a non-stable (or meta-stable) one, generally in presence of noise. Such interface separating the two phases develops many non-trivial geometric and statistical features during its time evolution. In two dimension a universal macroscopic behavior emerges, unifying many growth phenomena into a few universality classes, irrespective of their microscopic details. The most generic one, for stochastic growth rules, is the celebrated Kardar-Parisi-Zhang universality class. A prominent member of this class is the continuum KPZ equation [4]

$$
\partial_{t} h(x, t)=\nu \partial_{x}^{2} h(x, t)+\frac{\lambda_{0}}{2}\left(\partial_{x} h(x, t)\right)^{2}+\sqrt{D} \eta(x, t),
$$

which describes the motion of an interface of height $h(x, t)$ at point $x \in \mathbb{R}$ at time $t$, driven by a unit space-time white noise $\eta(x, t)$. In the past years many tools have been developed to solve this equation. Its solution is described at large times as a uniformly 
moving front with sub-leading $O\left(t^{1 / 3}\right)$ fluctuations, as $h(x, t)=v t+t^{\frac{1}{3}} \tilde{h}(x, t)+O(1)$, where $v$ is a parameter of the model and $\tilde{h}(x, t)$, the scaled height field, is a stochastic variable whose statistical properties become universal at large $t$. Numerous exact results were found $[5-16,18,24,25]$, as well as experimental and numerical results [26-28], showing the convergence at large time of the one-time probability distribution function $(\mathrm{PDF})$ of $\tilde{h}(x, t)$ towards a few universal distributions, the Tracy Widom (TW) distributions which also appear in random matrix theory. One approach is to map the solution of the KPZ equation to the free energy of a directed polymer (DP) in a random potential and then use standard replica methods to compute the integer moments, $\overline{Z^{n}}$, of the DP partition sum $[6,10-14]$. From these integer moments one extracts the probability distribution function (PDF) of the KPZ height field, a non-rigorous but generally correct approach. The average over the random potential leads to an effective interaction among the replicas, described by the attractive Lieb-Liniger (LL) Hamiltonian [29]. Therefore computing $\overline{Z^{n}}$ reduces to computing quantum transition amplitudes in imaginary time in the attractive LL model. Since this model can be solved via the Bethe ansatz, the method is called the replica Bethe ansatz (RBA). The ground state of the LL model is a single bound state containing all the particles (replicas), while excited states are obtained by splitting this bound state in several smaller ones [30]. To perform the calculation one expands over the complete set of eigenstates and re-sum the series. The contribution of the ground state is not sufficient to reproduce the long time limit of the KPZ equation (despite what intuition may suggest), and the other excited states need to be included in order to obtain the full statistics of the fluctuations at large times. This summation is not easy, but could be performed for one-time observables. For two-time observables however, such as the joint probability distribution (JPDF) of the scaled heights at two different times, $\tilde{h}\left(0, t_{1}\right)$ and $\tilde{h}\left(0, t_{2}\right)$, the calculation is very difficult $[33-37,40]$, and almost no practically useful results (i.e. which could be numerically evaluated and compared to numerical simulations and experiments) existed until recently.

In a recent work $[1,2]$ we obtained a formula for the tail of the two-time JPDF, for the droplet, or wedge, initial condition $h(x, t=0)=-w|x|$ in the large time limit. We started from the observation that the right tail of the one-time PDF of $\tilde{h}(x, t)$ at large $t$, i.e. of the TW distribution, can be well approximated from a proper treatment of the contribution of the LL ground state. We then conjectured that a similar property holds also for the two-time JPDF. That allowed us to perform a partial summation over states and obtain the following result. Consider the limit where both times $t_{1}, t_{2}$ are large but their ratio is kept finite $t_{2} / t_{1}=1+\Delta$. In that limit the joint cumulative probability distribution function (JCDF) becomes a function of $\Delta$ only

$$
\begin{aligned}
& \lim _{t_{1} \rightarrow \infty} \operatorname{Prob}\left(\tilde{h}\left(x_{1}, t_{1}\right)<\sigma_{1}, \tilde{h}\left(x_{2}, t_{1}(1+\Delta)\right)<\sigma_{2}\right)=\hat{\mathcal{P}}_{\Delta}\left(\sigma_{1}, \sigma_{2}\right), \\
& \hat{\mathcal{P}}_{\Delta}\left(\sigma_{1}, \sigma_{2}\right)=\mathcal{P}_{\Delta}\left(\sigma_{1}, \sigma\right) \quad, \quad \sigma_{2}=\frac{\sigma_{1}+\sigma \Delta^{1 / 3}}{(1+\Delta)^{1 / 3}},
\end{aligned}
$$

which we found more convenient to express (see second line) as a function of $\sigma$, which 
is associated to the scaled height difference (see below), a simple linear combination of $\sigma_{1}$ and $\sigma_{2}$. Our result was that for large positive $\sigma_{1}$ and any $\sigma, \Delta$

$$
\mathcal{P}_{\Delta}\left(\sigma_{1}, \sigma\right)=\mathcal{P}_{\Delta}^{(1)}\left(\sigma_{1}, \sigma\right)+O\left(e^{-\frac{8}{3} \sigma_{1}^{3 / 2}}\right),
$$

and we obtained an explicit formula for $\mathcal{P}_{\Delta}^{(1)}\left(\sigma_{1}, \sigma\right)$, see below.

The distribution $\mathcal{P}_{\Delta}^{(1)}$ was used to compute the conditional two-time covariance ratio

$$
C_{\Delta, \sigma_{c}}=\lim _{t_{1} \rightarrow \infty} \frac{\left\langle\tilde{h}\left(x, t_{1}\right) \tilde{h}\left(x, t_{1}(1+\Delta)\right)\right\rangle_{\tilde{h}\left(x, t_{1}\right)>\sigma_{c}}^{c}}{\left\langle\tilde{h}\left(x, t_{1}\right)^{2}\right\rangle_{\tilde{h}\left(x, t_{1}\right)>\sigma_{c}}^{c}},
$$

and to compare it with experimental and numerical results in $[1,2]$. We found excellent agreement for values of $\sigma_{c}>-1$, i.e. well beyond the naive range of validity, while the tail approximation starts to break down for smaller values of $\sigma_{c}$ (we recall that $\sigma_{c}=-1.7 \ldots$ is the mean of the TW distribution). This quantity shows how the system is aging during its time evolution and it is of theoretical [33-35,41] and experimental interest [31,32]. In particular its limit $\Delta \rightarrow \infty$ and $\sigma_{c} \rightarrow-\infty$ quantifies the so-called ergodicity breaking of the KPZ class [32]. In a separate RBA calculation, extending a framework based on Airy processes also discussed in [1,34] and valid for $\Delta \rightarrow+\infty$, it was obtained that for droplet initial conditions the full two-time covariance ratio does not decay to zero in the large $\Delta$ limit, but instead to a universal number [41]

$$
\lim _{\sigma_{c} \rightarrow-\infty} \lim _{\Delta \rightarrow \infty} C_{\Delta, \sigma_{c}}=0.623 \ldots
$$

in good agreement with previous experimental observations [43], and in excellent agreement with simultaneous numerical simulations [44]. The calculation of [41] also confirmed the result of $[1,2]$ but only in the regime where both $\sigma_{1}$ and $\Delta$ are simultaneously large (see also [42]).

Despite all the numerical and experimental evidence, a rigorous proof of the tail $\mathcal{P}_{\Delta}^{(1)}$ provided in [1,2] for arbitrary $\Delta$ was still lacking up to now. Recently an exact expression for the full two-time cumulative distribution $\hat{\mathcal{P}}_{\Delta}\left(\sigma_{1}, \sigma_{2}\right)$ was obtained by Johansson in [3]. It is obtained from a scaling limit of a calculation for a discrete DP model (last passage percolation), simpler than the one in [33]. It leads to a much more tractable expression than the one in [33], in fact a (relatively) simple one, as a contour integral of a matrix Fredholm determinant. Hence, we are now in position to check our expression for the tail, in the limit where $\sigma_{1}$ is large and positive.

In this paper we show that, by expanding the exact full two-time distribution of Ref. [3] at large and positive $\sigma_{1}$, the first order term of the expansion reproduces our result for the tail [1,2]. Our result therefore provides a non-trivial check of our expression for the tail and of the newly derived distribution [3]. 


\section{KPZ equation and results for the one-time statistics of the height}

While the large time results discussed here are expected to be valid for the whole KPZ class, independently of the details of the model, the explicit calculation performed in $[1,2]$ starts from the continuum KPZ equation (1). Using the units of space $x^{*}=\frac{(2 \nu)^{3}}{D \lambda_{0}^{2}}$, time $t^{*}=\frac{2(2 \nu)^{5}}{D^{2} \lambda_{0}^{4}}$ and height $h^{*}=\frac{2 \nu}{\lambda_{0}}$ it becomes

$$
\partial_{t} h(x, t)=\partial_{x}^{2} h(x, t)+\left(\partial_{x} h(x, t)\right)^{2}+\sqrt{2} \eta(x, t),
$$

with white noise $\overline{\eta(x, t) \eta\left(x^{\prime}, t^{\prime}\right)}=\delta\left(x-x^{\prime}\right) \delta\left(t-t^{\prime}\right)$. For the purpose of the calculation, the droplet IC was implemented using the so-called hard-wedge initial condition defined as $h_{w_{0}}(x, 0)=-w_{0}|x|+\ln \left(\frac{w_{0}}{2}\right)$ in the limit $w_{0} \rightarrow+\infty$, so that $\exp \left(h_{w_{0}}(x, 0)\right) \rightarrow \delta(x)$. Note that in the large time limit, the same universal results are expected for any finite $w_{0}>0$ or any other initial condition in the droplet class. At large time the KPZ field grows linearly in time with $O\left(t^{1 / 3}\right)$ fluctuations. The fluctuations at one space point (choosing here $x=0$ ) are governed by the Tracy Widom distribution associated to the Gaussian unitary ensemble (GUE). Its $\mathrm{PDF}$ is $f_{2}(\sigma)=F_{2}^{\prime}(\sigma)$, i.e. one has at large $t[5,6]$

$$
h(x=0, t)=-\frac{t}{12}+t^{1 / 3} \tilde{h}+o\left(t^{1 / 3}\right) \quad, \quad \operatorname{Prob}(\tilde{h}<\sigma)=F_{2}(\sigma),
$$

where $F_{2}(\sigma)$ is given by the Fredholm determinant

$$
F_{2}(\sigma)=\operatorname{Det}\left[I-P_{\sigma} K_{\mathrm{Ai}} P_{\sigma}\right]
$$

involving the Airy Kernel $K_{\mathrm{Ai}}$ :

$$
K_{\mathrm{Ai}}\left(v, v^{\prime}\right)=\int_{0}^{+\infty} d y \operatorname{Ai}(y+v) \operatorname{Ai}\left(y+v^{\prime}\right)=\frac{\operatorname{Ai}(v) \operatorname{Ai}^{\prime}\left(v^{\prime}\right)-\operatorname{Ai}^{\prime}(v) \operatorname{Ai}\left(v^{\prime}\right)}{v-v^{\prime}},
$$

where $P_{\sigma}(v)=\theta(v-\sigma)$ is the projector on $[\sigma,+\infty[$.

In [1] we introduced the tail approximation of the CDF of the GUE-TW distribution as the function

$$
F_{2}^{(1)}(\sigma) \equiv 1-\operatorname{Tr} P_{\sigma} K_{\mathrm{Ai}}=1-\int_{\sigma}^{+\infty} d v K_{\mathrm{Ai}}(v, v)
$$

which keeps only the first term in the series expansion of the Fredholm determinant, hence it captures the leading (stretched) exponential behavior for large and positive $\sigma$, $F_{2}^{(1)}(\sigma)-1=O\left(e^{-\frac{4}{3} \sigma^{3 / 2}}\right)$ and the corrections are terms $O\left(K_{\mathrm{Ai}}^{2}\right)$ containing products of four Airy functions and more, of higher (stretched) exponential order

$$
F_{2}(\sigma)=F_{2}^{(1)}(\sigma)+O\left(e^{-\frac{8}{3} \sigma^{3 / 2}}\right)
$$

which is the analog of (3) for the one-time distribution. As can be seen in Fig. 1 of [1] this approximation is very good (with error less than $10^{-3}$ ) for any $\sigma>-1$. This approximation also consists in keeping only single string states in the RBA method, leading to huge simplifications in the application of the method. 


\section{Tail of the two-time joint distribution from replica Bethe ansatz}

\subsection{Definitions and scaled variables}

Consider now the height at two different times $t=t_{1}$ and $t=t_{2}>t_{1}$, and two different space points $x=0$ and $x=X$, and denote

$$
H_{1} \equiv h\left(0, t_{1}\right)-v t_{1} \quad, \quad H_{2} \equiv h\left(X, t_{2}\right)-v t_{2} \quad, \quad H \equiv H_{21} \equiv H_{2}-H_{1},
$$

where $v=-1 / 12$ here. We have defined the difference of the two heights built over the time difference $t_{21} \equiv t_{2}-t_{1}$. In the large time limit, when both $t_{1}$ and $t_{2}$ are sent to $+\infty$, the relevant parameter characterizing the JPDF of the two heights will be the time difference rescaled by the earlier time, denoted as

$$
\Delta=\frac{t_{2}-t_{1}}{t_{1}}>0
$$

Let us start with $X=0$, the general case being discussed below. From the previous Section, we know that the two heights grow in time as $H_{1} \sim t_{1}^{1 / 3}$ and $H_{2} \sim t_{2}^{1 / 3}$, hence we introduce the rescaled heights $h_{1}, h_{2}$ and $h$, through

$$
H_{1}=t_{1}^{1 / 3} h_{1} \quad, \quad H_{2}=t_{2}^{1 / 3} h_{2} \quad, \quad H_{2}-H_{1}=\left(t_{2}-t_{1}\right)^{1 / 3} h=\Delta^{1 / 3} t_{1}^{1 / 3} h .
$$

Here $h_{1}, h_{2}, h$ are random variables. We note that

$$
h_{2}=\frac{h_{1}+h \Delta^{1 / 3}}{(1+\Delta)^{1 / 3}} \quad \Leftrightarrow h=h_{2}\left(1+\frac{1}{\Delta}\right)^{1 / 3}-h_{1} \Delta^{-1 / 3} .
$$

From the previous section, the PDF of $h_{1}$ is the GUE-TW, $f_{2}$, and similarly for $h_{2}$. The JCDF of $h_{1}$ and $h_{2}$ in the large time limit was defined in the introduction as

$$
\hat{\mathcal{P}}_{\Delta}\left(\sigma_{1}, \sigma_{2}\right)=\lim _{t_{1}, t_{2} \rightarrow+\infty, \frac{t_{1}-t_{2}}{t_{1}}=\Delta} \operatorname{Prob}\left(h_{1}<\sigma_{1}, h_{2}<\sigma_{2}\right),
$$

which depends only on $\Delta$. The variables which appear naturally in our result (and in Johansson's result, see below), are $\sigma_{1}$ and $\sigma=\sigma_{2}\left(1+\frac{1}{\Delta}\right)^{1 / 3}-\sigma_{1} \Delta^{-1 / 3}$, hence it is useful to define the function $\mathcal{P}_{\Delta}\left(\sigma_{1}, \sigma\right)$

$$
\mathcal{P}_{\Delta}\left(\sigma_{1}, \sigma\right)=\hat{\mathcal{P}}_{\Delta}\left(\sigma_{1}, \sigma_{2}=\frac{\sigma_{1}+\sigma \Delta^{1 / 3}}{(1+\Delta)^{1 / 3}}\right),
$$

which is simply (16) expressed in the variables $\sigma_{1}, \sigma$. Note that $\mathcal{P}_{\Delta}$ is not the JCDF associated to $h_{1}$ and $h$. However from it one can obtain the JPDF of $h_{1}$ and $h$ defined as

$$
P_{\Delta}\left(\sigma_{1}, \sigma\right)=\lim _{t_{1}, t_{2} \rightarrow+\infty, \frac{t_{1}-t_{2}}{t_{1}}=\Delta} \overline{\delta\left(h_{1}-\sigma_{1}\right) \delta(h-\sigma)}
$$

via the relation

$$
P_{\Delta}\left(\sigma_{1}, \sigma\right)=\left(\partial_{\sigma_{1}} \partial_{\sigma}-\Delta^{-1 / 3} \partial_{\sigma}^{2}\right) \mathcal{P}_{\Delta}\left(\sigma_{1}, \sigma\right),
$$

whose derivation is a simple change of variable detailed in Appendix A. 


\subsection{Tail of the two-time distribution}

The calculation of the full distribution (16), (17), (18) using the RBA method poses a formidable challenge via the replica method (a valid attempt was also conducted in [35-37] but it turned out to be incorrect, see [1]). The difficulty comes from the summation over two sets of eigenfunctions of the LL model, and the complicated expression for the so-called form factors $[38,39]$. In $[1,2]$ using a partial summation, where one of the set is restricted to the single string states of the Lieb-Liniger model, we were able to derive a relatively simple expression for the tail approximation $\mathcal{P}_{\Delta}^{(1)}\left(\sigma_{1}, \sigma\right)$ of the cumulative distribution (which satisfies (3))

$\mathcal{P}^{(1)}\left(\sigma_{1}, \sigma\right)=1+\left(F_{2}^{(1)}\left(\sigma_{2}\right)-1\right)+\left(F_{2}(\sigma) \operatorname{Tr}\left[\Delta^{1 / 3} P_{\sigma} K_{\sigma_{1}}^{\Delta} P_{\sigma}\left(I-P_{\sigma} K_{\mathrm{Ai}} P_{\sigma}\right)^{-1}-P_{\sigma_{1}} K_{\mathrm{Ai}}\right]\right)$,

with $\ddagger \sigma_{2}=\frac{\sigma_{1}+\sigma \Delta^{1 / 3}}{(1+\Delta)^{1 / 3}}$, expressed in terms of the Airy kernel, as well as of a novel kernel

$$
K_{\sigma_{1}}^{\Delta}(u, v)=\int_{0}^{\infty} d y_{1} d y_{2} \operatorname{Ai}\left(-y_{1}+u\right) K_{\mathrm{Ai}}\left(y_{1} \Delta^{1 / 3}+\sigma_{1}, y_{2} \Delta^{1 / 3}+\sigma_{1}\right) \operatorname{Ai}\left(-y_{2}+v\right) \text {, }
$$

where we recall that $F_{2}(\sigma)$ and $F_{2}^{(1)}\left(\sigma_{1}\right)$ are respectively the GUE-TW CDF and its tail approximation, given respectively by $(8)$ and (10).

The corresponding tail approximation JPDF was displayed in [1] Eq. 23 as

$$
P_{\Delta}^{(1)}\left(\sigma_{1}, \sigma\right)=\left(\partial_{\sigma_{1}} \partial_{\sigma}-\Delta^{-1 / 3} \partial_{\sigma}^{2}\right) \mathcal{P}_{\Delta}^{(1)}\left(\sigma_{1}, \sigma\right) .
$$

Our result for the JPDF satisfies two important properties. In the limit of infinite time difference $t_{2} / t_{1} \rightarrow+\infty$ (which corresponds to the limit $\Delta \rightarrow+\infty$ ), it converges to the product of two GUE-TW distributions

$$
\lim _{\Delta \rightarrow \infty} P_{\Delta}^{(1)}\left(\sigma_{1}, \sigma\right)=F_{2}^{(1) \prime}\left(\sigma_{1}\right) F_{2}^{\prime}(\sigma),
$$

and in the limit of small (scaled) time separation $\left(t_{2}-t_{1}\right) / t_{1} \ll 1$ (which translates into $\left.\Delta \rightarrow 0^{+}\right)$it also decouples as follows

$$
\lim _{\Delta \rightarrow 0} P_{\Delta}^{(1)}\left(\sigma_{1}, \sigma\right)=F_{2}^{(1) \prime}\left(\sigma_{1}\right) F_{0}^{\prime}(\sigma)
$$

where $F_{0}(\sigma)$ is the Baik-Rains (BR) cumulative distribution $[16,17]$ which governs the stationary growth profile in the infinite time limit.

\section{Johansson's result for the full two-time joint probability distribution}

Here we report Johansson's recent result [3] in our present notations. We focus here on the joint distribution for the two heights at the same position $X=0$. There the $\ddagger$ The $\operatorname{CDF} \hat{\mathcal{P}}=\mathcal{P}$ is $g_{\Delta}$ in Eq. (75) in [1], where we corrected a misprint (immaterial for the main 
following functions are defined

$$
\begin{gathered}
S_{1}(x, y)=-\Delta^{-1 / 3} e^{(y-x) \delta} \int_{0}^{\infty} d s K_{\mathrm{Ai}}\left(\sigma_{1}-s, \sigma_{1}-x\right) K_{\mathrm{Ai}}\left(\sigma+s \Delta^{-1 / 3}, \sigma+y \Delta^{-1 / 3}\right) \\
T_{1}(x, y)=\Delta^{-1 / 3} e^{(y-x) \delta} \int_{-\infty}^{0} d s K_{\mathrm{Ai}}\left(\sigma_{1}-s, \sigma_{1}-x\right) K_{\mathrm{Ai}}\left(\sigma+s \Delta^{-1 / 3}, \sigma+y \Delta^{-1 / 3}\right) \\
S_{2}(x, y)=\Delta^{-1 / 3} e^{\delta(y-x)} K_{\mathrm{Ai}}\left(\sigma+x \Delta^{-1 / 3}, \sigma+y \Delta^{-1 / 3}\right) \\
S_{3}(x, y)=e^{\delta(y-x)} K_{\mathrm{Ai}}\left(\sigma_{1}-x, \sigma_{1}-y\right)
\end{gathered}
$$

Notice that the regulator $\delta>0$ can be set to $0^{+}$. Moreover the following composite kernels are introduced

$$
\begin{aligned}
& S(x, y)=S_{1}(x, y)+\theta(x) S_{2}(x, y)-\theta(-y) S_{3}(x, y), \\
& T(x, y)=-T_{1}(x, y)-\theta(x) S_{2}(x, y)+\theta(-y) S_{3}(x, y) .
\end{aligned}
$$

The full kernel is then given by, defining $R_{u}(x, y)=S(x, y)+u^{-1} T(x, y)$

$$
K_{u}=\left(\begin{array}{cc}
P^{-} R_{u} P^{-} & P^{-} R_{u} P^{+} \\
u P^{+} R_{u} P^{-} & u P^{+} R_{u} P^{+}
\end{array}\right),
$$

where we introduced the projectors $P^{+}$on the interval $[0, \infty)$ and $P^{-}$on the interval $(-\infty, 0]$. Then theorem 2.1 plus the formula (15) in [3] shows that the joint probability distribution at two different times can be written as the contour integral of a Fredholm determinant

$$
\begin{aligned}
\hat{\mathcal{P}}_{\Delta}\left(\sigma_{1}, \sigma_{2}\right) & =\lim _{t_{1}, t_{2} \rightarrow+\infty, \frac{t_{1}-t_{2}}{t_{1}}=\Delta} \operatorname{Prob}\left(h_{1}<\sigma_{1}, h_{2}<\sigma_{2}\right) \\
& =\frac{1}{2 \pi i} \int_{R_{1}} \frac{d u}{u-1} \operatorname{Det}\left(1+K_{u}\right) \\
& =\mathcal{P}\left(\sigma_{1}, \sigma\right),
\end{aligned}
$$

with $R_{1}$ a circle of radius larger than 1 . As expressed by the last line, this formula, quite remarkably, is expressed naturally as a function of of $\sigma_{1}$ and $\sigma=\sigma_{2}\left(1+\frac{1}{\Delta}\right)^{1 / 3}-\sigma_{1} \Delta^{-1 / 3}$, a property already found in our tail result, as discussed above.

Before embarking on calculations it is useful to define the following functions, defined via a similarity transformation

$$
S_{1}(x, y)=\Delta^{-1 / 3} \tilde{S}_{1}\left(x \Delta^{-1 / 3}, y \Delta^{-1 / 3}\right),
$$

and the same definition (and tilde notation) for $S_{2}$ and $T_{1}$, leading to

$$
\begin{aligned}
& \tilde{S}_{1}(x, y)=-\Delta^{1 / 3} \int_{0}^{\infty} d s K_{\mathrm{Ai}}\left(\sigma_{1}-s \Delta^{1 / 3}, \sigma_{1}-x \Delta^{1 / 3}\right) K_{\mathrm{Ai}}(\sigma+s, \sigma+y), \\
& \tilde{T}_{1}(x, y)=\Delta^{1 / 3} \int_{-\infty}^{0} d s K_{\mathrm{Ai}}\left(\sigma_{1}-s \Delta^{1 / 3}, \sigma_{1}-x \Delta^{1 / 3}\right) K_{\mathrm{Ai}}(\sigma+s, \sigma+y), \\
& \tilde{S}_{2}(x, y)=K_{\mathrm{Ai}}(\sigma+x, \sigma+y)
\end{aligned}
$$


where we have also changed $s \rightarrow \Delta^{1 / 3} s$ in the integrals. Since similarity transformations do not change the values of traces and determinants, they will be useful below.

\section{Tail of Johansson's two-time distribution}

In this section we will expand Johansson's result (32) for the JCDF for large positive values of $\sigma_{1}$ at a given value of $\sigma$.

\subsection{Limit $\sigma_{1} \rightarrow+\infty$ of Johansson's formula}

We first consider the limit $\sigma_{1} \rightarrow+\infty$ of the JCDF (32). Since $\sigma$ is fixed it implies also $\sigma_{2} \rightarrow+\infty$. Hence we should simply obtain

$$
\lim _{\sigma_{1} \rightarrow+\infty} \hat{\mathcal{P}}\left(\sigma_{1}, \sigma\right)=1
$$

a simple check on the formula. Due to the presence of Airy kernels with $\sigma_{1}$ in their argument, in this limit we have $S_{1}, T_{1}, S_{3} \rightarrow 0$. Therefore neglecting sub-leading corrections in $\operatorname{Ai}\left(\sigma_{1}+\ldots\right) \sim e^{-\frac{2}{3} \sigma_{1}^{3 / 2}}$ at large $\sigma_{1}$, we obtain

$$
\begin{aligned}
& S(x, y) \rightarrow \theta(x) S_{2}(x, y), \\
& T(x, y) \rightarrow \theta(x) S_{2}(x, y), \\
& R_{u}(x, y) \rightarrow \theta(x) S_{2}(x, y)\left(1-u^{-1}\right) .
\end{aligned}
$$

This implies that the Fredholm determinant in this limit is given by

$$
\begin{aligned}
\operatorname{Det}\left(1+K_{u}\right) & \rightarrow \operatorname{Det}\left(\begin{array}{cc}
1 & 0 \\
u P^{+} R_{u} P^{-} & 1+u P^{+} R_{u} P^{+}
\end{array}\right) \\
& =\operatorname{Det}\left(1-(1-u) P^{+} S_{2} P^{+}\right) \\
& =\operatorname{Det}\left(1-(1-u) P^{+} \tilde{S}_{2} P^{+}\right) \\
& =\operatorname{Det}\left(1+(u-1) P_{\sigma} K_{\mathrm{Ai}} P_{\sigma}\right),
\end{aligned}
$$

where we have used the similarity transformation (35) for $S_{2}$ and the expression (38) for $\tilde{S}_{2}$, which is simply the Airy kernel. To perform the integration over $u$ it is convenient to expand the Fredholm determinant in powers of its trace, namely

$$
\operatorname{Det}(1+f(u) A)=1+\sum_{n=1}^{\infty} \frac{(f(u))^{n}}{n !}\left(\prod_{j=1}^{n} \int d x_{j}\right) \operatorname{det}_{i, j=1}^{n} A\left(x_{i}, x_{j}\right)
$$

Therefore, pulling out the factor $(u-1)^{n}$ at each order, and using the identity

$$
\frac{1}{2 \pi i} \int_{R_{1}} \frac{d u}{u-1}(u-1)^{n}=\delta_{n, 0} \quad \forall n \geq 0
$$

we obtain the expected result (39). 


\subsection{Large $\sigma_{1}$ : leading tail contribution}

We now obtain the leading correction for large $\sigma_{1}$ at fixed $\sigma$. Let us first rewrite (29) in the more compact form using the projectors

$$
S=S_{1}+P^{+} S_{2}-S_{3} P^{-} \quad, \quad T=-T_{1}-P^{+} S_{2}+S_{3} P^{-},
$$

which leads to

$\operatorname{Det}\left(I+K_{u}\right)$

$$
=\operatorname{Det}\left(\begin{array}{cc}
I+P^{-}\left(S_{1}-S_{3}+u^{-1}\left(-T_{1}+S_{3}\right)\right) P^{-} & P^{-}\left(S_{1}-u^{-1} T_{1}\right) P^{+} \\
P^{+}\left(u\left(S_{1}+S_{2}-S_{3}\right)+\left(-T_{1}-S_{2}+S_{3}\right)\right) P^{-} & I+P^{+}\left(u\left(S_{1}+S_{2}\right)+\left(-T_{1}-S_{2}\right)\right) P^{+}
\end{array}\right) .
$$

In the large positive $\sigma_{1}$ limit, we can introduce a natural small parameter, namely $\epsilon \sim \operatorname{Ai}\left(\sigma_{1}\right)^{2}$. Then one has

$$
S_{1} \sim T_{1} \sim S_{3} \sim \epsilon \quad, \quad S_{2} \sim O(1)
$$

We will thus write the kernel as a sum of a $O(1)$ piece and a piece $\sim \epsilon$ as

$$
I+K_{u}=\left(\begin{array}{cc}
I & 0 \\
C_{0} & D_{0}
\end{array}\right)+\left(\begin{array}{cc}
A_{1} & B_{1} \\
C_{1} & D_{1}
\end{array}\right)=M_{0}+M_{1}
$$

where we have defined

$$
C_{0}=(u-1) P^{+} S_{2} P^{-} \quad, \quad D_{0}=I+(u-1) P^{+} S_{2} P^{+},
$$

and the perturbations which are $O(\epsilon)$

$$
\begin{aligned}
A_{1} & =P^{-}\left(S_{1}-\frac{1}{u} T_{1}+S_{3} \frac{1-u}{u}\right) P^{-} \\
B_{1} & =P^{-}\left(S_{1}-\frac{1}{u} T_{1}\right) P^{+} \\
C_{1} & =P^{+}\left(u\left(S_{1}-S_{3}\right)+\left(S_{3}-T_{1}\right)\right) P^{-} \\
D_{1} & =P^{+}\left(u S_{1}-T_{1}\right) P^{+} .
\end{aligned}
$$

Using the expansion of the determinant around a fixed matrix

$\operatorname{Det}\left(M_{0}+M_{1}\right)=\operatorname{Det} M_{0}\left(1+\operatorname{Tr} M_{1} M_{0}^{-1}+\frac{1}{2}\left(\left(\operatorname{Tr} M_{1} M_{0}^{-1}\right)^{2}-\operatorname{Tr}\left(M_{1} M_{0}^{-1}\right)^{2}\right)+O\left(M_{1}^{3}\right)\right)$, and the inversion formula $\left(\begin{array}{cc}I & 0 \\ C_{0} & D_{0}\end{array}\right)^{-1}=\left(\begin{array}{cc}I & 0 \\ -D_{0}^{-1} C_{0} & D_{0}^{-1}\end{array}\right)$, we obtain to $O(\epsilon)$

$$
\operatorname{Det}\left(1+K_{u}\right)=\operatorname{Det} D_{0}\left(1+\operatorname{Tr} D_{1} D_{0}^{-1}+\operatorname{Tr} A_{1}-\operatorname{Tr} B_{1} D_{0}^{-1} C_{0}\right)+O\left(\epsilon^{2}\right),
$$

where the trace of an operator is defined as usual as

$$
\operatorname{Tr} A=\int d x A(x, x)=\int d y \tilde{A}(y, y) \quad, \quad A\left(x, x^{\prime}\right)=\lambda \tilde{A}\left(\lambda x, \lambda x^{\prime}\right)
$$


and is invariant under the similarity transform $y=\lambda x$, a property used below.

We can now carry the integration over $u$ of (56) (since all operators there are $u$-dependent) as in (32). It turns out that one only needs the formula

$$
\frac{1}{2 \pi i} \int_{R_{1}} \frac{d u}{u-1}\left(p_{0}(u)+\frac{1}{u} p_{1}(u)\right)=p_{0}(0)+p_{1}(0)-p_{1}(1)
$$

where $p_{0}, p_{1}$ are arbitrary analytic functions in $u$. Indeed $A_{1}, B_{1}, D_{1}, C_{0}$ are of this form with $p_{0}, p_{1}$ simple polynomials. Note that possible poles in the inverse $D_{0}^{-1}=$ $\left(I+(u-1) P^{+} S_{2} P^{+}\right)^{-1}$ would be canceled by the determinant $\operatorname{Det} D_{0}$ which always appear in factor. Hence we can expand Det $D_{0}$ and $D_{0}^{-1}$ to powers of $(u-1)$ and formally treat them as polynomials.

Using (58) to integrate over $u$, we obtain from (56) and (32) for the various terms

$$
\begin{array}{ll}
\frac{1}{2 \pi i} \int_{R_{1}} \frac{d u}{u-1} \operatorname{Det} D_{0} \operatorname{Tr} D_{1} D_{0}^{-1} & =\operatorname{Tr} P^{+}\left(S_{1}-T_{1}\right) P^{+}, \\
\frac{1}{2 \pi i} \int_{R_{1}} \frac{d u}{u-1} \operatorname{Det} D_{0} \operatorname{Tr} A_{1} & =\operatorname{Tr} P^{-}\left(S_{1}-T_{1}\right) P^{-}-F_{2}(\sigma) \operatorname{Tr} P^{-}\left(S_{3}-T_{1}\right) P^{-}, . \\
\frac{1}{2 \pi i} \int_{R_{1}} \frac{d u}{u-1} \operatorname{Det} D_{0} \operatorname{Tr} B_{1} D_{0}^{-1} C_{0} & =-F_{2}(\sigma) \operatorname{Tr} P^{-} T_{1} P^{+}\left[1-P^{+} S_{2} P^{+}\right]^{-1} P^{+} S_{2} P^{-},
\end{array}
$$

where we used that $\operatorname{Det}\left(1-P^{+} S_{2} P^{+}\right)=F_{2}(\sigma)$. Putting everything together and simplifying we finally obtain

$$
\begin{aligned}
\mathcal{P}_{\Delta}\left(\sigma_{1}, \sigma\right) & =1+\operatorname{Tr}\left(S_{1}-T_{1}\right)+F_{2}(\sigma) \operatorname{Tr} P^{-}\left(T_{1}-S_{3}\right), \\
& +F_{2}(\sigma) \operatorname{Tr} P^{-} T_{1} P^{+}\left(1-P^{+} S_{2} P^{+}\right)^{-1} P^{+} S_{2} \quad+O\left(\epsilon^{2}\right) .
\end{aligned}
$$

\section{Exact agreement between the two distributions in the large $\sigma_{1}$ limit}

\subsection{Summary}

Here we show that the two tails exactly agree. Let us recall our previous result for the tail of equation (20). Using that $\left(I-P_{\sigma} K_{\mathrm{Ai}} P_{\sigma}\right)^{-1}=I+\left(I-P_{\sigma} K_{\mathrm{Ai}} P_{\sigma}\right)^{-1} P_{\sigma} K_{\mathrm{Ai}} P_{\sigma}$ it can be rewritten as

$$
\begin{aligned}
\mathcal{P}_{\Delta}^{(1)}\left(\sigma_{1}, \sigma\right) & =1+\left(F_{2}^{(1)}\left(\sigma_{2}\right)-1\right)-F_{2}(\sigma) \operatorname{Tr} P_{\sigma_{1}} K_{\mathrm{Ai}}+F_{2}(\sigma) \Delta^{1 / 3} \operatorname{Tr} P_{\sigma} K_{\sigma_{1}}^{\Delta} \\
& +F_{2}(\sigma) \Delta^{1 / 3} \operatorname{Tr} P_{\sigma} K_{\mathrm{Ai}} P_{\sigma} K_{\sigma_{1}}^{\Delta} P_{\sigma}\left(I-P_{\sigma} K_{\mathrm{Ai}} P_{\sigma}\right)^{-1} .
\end{aligned}
$$

We now compare Johansson's equation (60) with our previous result (61). As we show below one has

$$
\begin{gathered}
\operatorname{Tr}\left(S_{1}-T_{1}\right)=F_{2}^{(1)}\left(\sigma_{2}\right)-1 \\
-\operatorname{Tr} P^{-} S_{3}=-\operatorname{Tr} P_{\sigma_{1}} K_{\mathrm{Ai}}
\end{gathered}
$$

result Eq. (23) there), together with the tail approximation Eq. (139) there. 


$$
\operatorname{Tr} P^{-} T_{1}=\Delta^{1 / 3} \operatorname{Tr} P_{\sigma} K_{\sigma_{1}}^{\Delta}
$$

$\operatorname{Tr} P^{-} T_{1} P^{+}\left(1-P^{+} S_{2} P^{+}\right)^{-1} P^{+} S_{2}=\Delta^{1 / 3} \operatorname{Tr} P_{\sigma} K_{\mathrm{Ai}} P_{\sigma} K_{\sigma_{1}}^{\Delta} P_{\sigma}\left(I-P_{\sigma} K_{\mathrm{Ai}} P_{\sigma}\right)^{-1}$.

Hence the two formula (20) and (60) are identical. The tail obtained that we obtained in Ref. [1,2] is thus confirmed by the exact result of Johansson [3].

\subsection{Proof of the above relations}

We now show the above relations. We recall the definition (21) of the kernel $K_{\sigma_{1}}^{\Delta}$. Since it involves $\Delta^{1 / 3}$ rather that $\Delta^{-1 / 3}$, it is more convenient to use the similarity transformed functions $\tilde{S}_{1}, \tilde{S}_{2}, \tilde{T}_{1}$ defined in (36) and use the invariance of the trace under the similarity transformation (35).

Proof of relation (62). For notational simplicity we use here and below notations such as $\int_{s} \equiv \int_{-\infty}^{+\infty} d s, \int_{s>0} \equiv \int_{0}^{+\infty} d s, \int_{s, x>0} \equiv \int_{0}^{+\infty} d s \int_{0}^{+\infty} d x, \int_{s>0, x} \equiv \int_{0}^{+\infty} d s \int_{-\infty}^{+\infty} d x$ etc. One has

$$
\begin{aligned}
& \operatorname{Tr}\left(S_{1}-T_{1}\right)=\operatorname{Tr}\left(\tilde{S}_{1}-\tilde{T}_{1}\right)=-\int_{s, x} K_{\mathrm{Ai}}\left(\sigma_{1}-s \Delta^{1 / 3}, \sigma_{1}-x \Delta^{1 / 3}\right) K_{\mathrm{Ai}}(\sigma+s, \sigma+x) \\
& =-\int_{z_{1}, z_{2}>0, s, x} \operatorname{Ai}\left(\sigma_{1}-s \Delta^{1 / 3}+z_{1}\right) \operatorname{Ai}\left(\sigma_{1}-x \Delta^{1 / 3}+z_{1}\right) \operatorname{Ai}\left(\sigma+s+z_{2}\right) \operatorname{Ai}\left(\sigma+x+z_{2}\right) .
\end{aligned}
$$

Now we use twice the identity

$$
\int_{s} \operatorname{Ai}\left(a-s \Delta^{1 / 3}\right) \operatorname{Ai}(b+s)=\frac{1}{(1+\Delta)^{1 / 3}} \operatorname{Ai}\left(\frac{a+\Delta^{1 / 3} b}{(1+\Delta)^{1 / 3}}\right),
$$

and we obtain

$$
\begin{aligned}
\operatorname{Tr}\left(S_{1}-T_{1}\right) & =-\frac{1}{(1+\Delta)^{2 / 3}} \int_{z_{1}, z_{2}>0}\left[\operatorname{Ai}\left(\frac{\sigma_{1}+z_{1}+\Delta^{1 / 3}\left(\sigma+z_{2}\right)}{(1+\Delta)^{1 / 3}}\right)\right]^{2} \\
& =-\int_{z_{1}, z_{2}>0}\left[\operatorname{Ai}\left(z_{1}+z_{2}+\sigma_{2}\right)\right]^{2}=-\operatorname{Tr} P_{\sigma_{2}} K_{\mathrm{Ai}}=F_{2}^{(1)}\left(\sigma_{2}\right)-1
\end{aligned}
$$

where $\sigma_{2}=\frac{\sigma_{1}+\Delta^{1 / 3} \sigma}{(1+\Delta)^{1 / 3}}$ and we have changed $z_{1,2} \rightarrow(1+\Delta)^{1 / 3} z_{1,2}$ in the integrals.

Proof of relation (63). By definition from (28).

Proof of relation (64). From (37) one has

$$
\operatorname{Tr} P^{-} T_{1}=\operatorname{Tr} P^{-} \tilde{T}_{1}=\Delta^{1 / 3} \int_{x, s>0} K_{\mathrm{Ai}}\left(\sigma_{1}+\Delta^{1 / 3} s, \sigma_{1}+\Delta^{1 / 3} x\right) K_{\mathrm{Ai}}(\sigma-s, \sigma-x),
$$


where we have changed $(x, s) \rightarrow(-x,-s)$. On the other hand, from the definition $(21)$, our result is

$$
\begin{aligned}
\Delta^{1 / 3} \operatorname{Tr} P_{\sigma} K_{\sigma_{1}}^{\Delta} & =\Delta^{1 / 3} \int_{0}^{\infty} d v \int_{0}^{\infty} d y_{1} d y_{2} \operatorname{Ai}\left(-y_{1}+v+\sigma\right) \\
& \times K_{\mathrm{Ai}}\left(y_{1} \Delta^{1 / 3}+\sigma_{1}, y_{2} \Delta^{1 / 3}+\sigma_{1}\right) \operatorname{Ai}\left(-y_{2}+v+\sigma\right) \\
& =\Delta^{1 / 3} \int_{0}^{\infty} d y_{1} d y_{2} K_{\mathrm{Ai}}\left(y_{1} \Delta^{1 / 3}+\sigma_{1}, y_{2} \Delta^{1 / 3}+\sigma_{1}\right) K_{\mathrm{Ai}}\left(-y_{1}+\sigma,-y_{2}+\sigma\right)
\end{aligned}
$$

and therefore they are the same.

Proof of relation (65). We will prove the equivalent relation

$$
\operatorname{Tr} P^{+} \tilde{S}_{2} P^{-} \tilde{T}_{1} P^{+}\left(1-P^{+} \tilde{S}_{2} P^{+}\right)^{-1}=\Delta^{1 / 3} \operatorname{Tr} P_{\sigma} K_{\mathrm{Ai}} P_{\sigma} K_{\sigma_{1}}^{\Delta} P_{\sigma}\left(I-P_{\sigma} K_{\mathrm{Ai}} P_{\sigma}\right)^{-1} .
$$

This calculation is not trivial due to the presence of the inverse. Given a generic operator $A$, we use the definition of its inverse

$$
(I-A)^{-1}=\sum_{n=0}^{\infty} A^{n}=I+A+A^{2}+\ldots
$$

It is thus sufficient that for any $n \geq 0$

$$
\operatorname{Tr} P^{+} \tilde{S}_{2} P^{-} \tilde{T}_{1}\left(P^{+} \tilde{S}_{2}\right)^{n}=\Delta^{1 / 3} \operatorname{Tr} P_{\sigma} K_{\mathrm{Ai}} P_{\sigma} K_{\sigma_{1}}^{\Delta}\left(P_{\sigma} K_{\mathrm{Ai}}\right)^{n},
$$

which we now prove. We start by showing the basic identity

$$
\int_{y_{1}>0} \operatorname{Ai}\left(\sigma+z-y_{1}\right) \tilde{T}_{1}\left(-y_{1}, y\right)=\Delta^{1 / 3} \int_{p_{1}>0} K_{\sigma_{1}}^{\Delta}\left(\sigma+z, \sigma+p_{1}\right) \operatorname{Ai}\left(\sigma+p_{1}+y\right) .
$$

From the definition (37) we have (setting $s=-y_{2}$ )

$$
\begin{aligned}
& \Delta^{-1 / 3} \int_{y_{1}>0} \operatorname{Ai}\left(\sigma+z-y_{1}\right) \tilde{T}_{1}\left(-y_{1}, y\right) \\
& =\int_{y_{1}, y_{2}>0} \operatorname{Ai}\left(\sigma+z-y_{1}\right) K_{\mathrm{Ai}}\left(\sigma_{1}+y_{2} \Delta^{1 / 3}, \sigma_{1}+y_{1} \Delta^{1 / 3}\right) K_{\mathrm{Ai}}\left(\sigma-y_{2}, \sigma+y\right) \\
& =\int_{p_{1}, y_{1}, y_{2}>0} \operatorname{Ai}\left(\sigma+z-y_{1}\right) K_{\mathrm{Ai}}\left(\sigma_{1}+y_{2} \Delta^{1 / 3}, \sigma_{1}+y_{1} \Delta^{1 / 3}\right) \operatorname{Ai}\left(\sigma-y_{2}+p_{1}\right) \operatorname{Ai}\left(\sigma+y+p_{1}\right) \\
& =\int_{p_{1}>0} K_{\sigma_{1}}^{\Delta}\left(\sigma+z, \sigma+p_{1}\right) \operatorname{Ai}\left(\sigma+p_{1}+y\right)
\end{aligned}
$$

using the definition (21), which shows (73). Multiplying the l.h.s of (73) by $\operatorname{Ai}(\sigma+z+x)$ and integrating over $z>0$ we obtain a second useful identity

$$
\begin{aligned}
& \left(\tilde{S}_{2} P^{-} \tilde{T}_{1}\right)(x, y)=\int_{y_{1}>0} K_{\mathrm{Ai}}\left(\sigma+x, \sigma-y_{1}\right) \tilde{T}_{1}\left(-y_{1}, y\right) \\
& =\Delta^{1 / 3} \int_{z>0, p_{1}>0} \operatorname{Ai}(\sigma+z+x) K_{\sigma_{1}}^{\Delta}\left(\sigma+z, \sigma+p_{1}\right) \operatorname{Ai}\left(\sigma+p_{1}+y\right) .
\end{aligned}
$$


This can be rewritten more compactly, using the ket-bra notation for vectors and operators (as in quantum mechanics, i.e. $\langle x \mid \phi\rangle=\phi(x),\langle\psi|O| \phi\rangle=\int_{x y} \psi(x) O(x, y) \phi(y)$ etc..) as

$$
\left(\tilde{S}_{2} P^{-} \tilde{T}_{1}\right)(x, y)=\Delta^{1 / 3}\left\langle\mathrm{Ai}_{\sigma+x}\left|P^{+} K_{\sigma_{1}, \sigma}^{\Delta} P^{+}\right| \mathrm{Ai}_{\sigma+y}\right\rangle,
$$

where here and below we denote

$$
\operatorname{Ai}_{\sigma}(u)=\operatorname{Ai}(\sigma+u) \quad, \quad K_{\sigma_{1}, \sigma}^{\Delta}(u, v)=K_{\sigma_{1}}^{\Delta}(\sigma+u, \sigma+v) .
$$

Let us show (72) for $n=0$. One has

$$
\begin{aligned}
\operatorname{Tr} P^{+} \tilde{S}_{2} P^{-} \tilde{T}_{1} & =\int_{x>0}\left(\tilde{S}_{2} P^{-} \tilde{T}_{1}\right)(x, x)=\Delta^{1 / 3} \int_{x>0}\left\langle\mathrm{Ai}_{\sigma+x}\left|P^{+} K_{\sigma_{1}, \sigma}^{\Delta} P^{+}\right| \mathrm{Ai}_{\sigma+x}\right\rangle \\
& =\Delta^{1 / 3} \operatorname{Tr} P^{+} K_{\mathrm{Ai}, \sigma} P^{+} K_{\sigma_{1}, \sigma}^{\Delta}=\Delta^{1 / 3} \operatorname{Tr} P_{\sigma} K_{\mathrm{Ai}} P_{\sigma} K_{\sigma_{1}}^{\Delta},
\end{aligned}
$$

where we have used the definition of the Airy kernel

$$
K_{\mathrm{Ai}, \sigma}=\int_{z>0}\left|\mathrm{Ai}_{\sigma+z}\right\rangle\left\langle\mathrm{Ai}_{\sigma+z}\right| \Leftrightarrow K_{\mathrm{Ai}, \sigma}(x, y)=\left\langle x\left|K_{\mathrm{Ai}, \sigma}\right| y\right\rangle=\int_{z>0} \operatorname{Ai}(\sigma+z+x) \operatorname{Ai}(\sigma+z+y) .
$$

We now turn to arbitrary $n$. One has

$$
\operatorname{Tr} P^{+} \tilde{S}_{2} P^{-} \tilde{T}_{1}\left(P^{+} \tilde{S}_{2}\right)^{n}=\int_{x, y>0}\left(\tilde{S}_{2} P^{-} \tilde{T}_{1}\right)(x, y)\left(P^{+} \tilde{S}_{2}\right)^{n}(y, x) .
$$

We now use the second equivalent definition of the Airy kernel

$$
\tilde{S}_{2}(x, y)=K_{\mathrm{Ai}, \sigma}(x, y)=\left\langle\mathrm{Ai}_{\sigma+x}\left|P^{+}\right| \mathrm{Ai}_{\sigma+y}\right\rangle,
$$

which leads to

$$
\begin{aligned}
& \left(P^{+} \tilde{S}_{2}\right)^{n}(y, x) \\
& =P^{+} \int_{z_{1}, z_{2}, \ldots, z_{n-1}>0}\left\langle\mathrm{Ai}_{\sigma+y}\left|P^{+}\right| \mathrm{Ai}_{\sigma+z_{1}}\right\rangle\left\langle\mathrm{Ai}_{\sigma+z_{1}}\left|P^{+}\right| \mathrm{Ai}_{\sigma+z_{2}}\right\rangle \cdots\left\langle\mathrm{Ai}_{\sigma+z_{n-1}}\left|P^{+}\right| \mathrm{Ai}_{\sigma+x}\right\rangle .
\end{aligned}
$$

Inserting (78) and (85) in (83) we obtain

$$
\begin{aligned}
\operatorname{Tr} P^{+} \tilde{S}_{2} P^{-} \tilde{T}_{1}\left(P^{+} \tilde{S}_{2}\right)^{n} & =\Delta^{1 / 3} \int_{x, y, z_{1}, z_{2}, \ldots, z_{n-1}>0}\left\langle\mathrm{Ai}_{\sigma+x}\left|P^{+} K_{\sigma_{1}, \sigma}^{\Delta} P^{+}\right| \mathrm{Ai}_{\sigma+y}\right\rangle \\
& \times\left\langle\mathrm{Ai}_{\sigma+y}\left|P^{+}\right| \mathrm{Ai}_{\sigma+z_{1}}\right\rangle\left\langle\mathrm{Ai}_{\sigma+z_{1}}\left|P^{+}\right| \mathrm{Ai}_{\sigma+z_{2}}\right\rangle \cdots\left\langle\mathrm{Ai}_{\sigma+z_{n-1}}\left|P^{+}\right| \mathrm{Ai}_{\sigma+x}\right\rangle \\
& =\Delta^{1 / 3} \operatorname{Tr} P^{+} K_{\mathrm{Ai}, \sigma} P^{+} K_{\sigma_{1}, \sigma}^{\Delta}\left(P^{+} K_{\mathrm{Ai}, \sigma}\right)^{n} \\
& =\Delta^{1 / 3} \operatorname{Tr} P_{\sigma} K_{\mathrm{Ai}} P_{\sigma} K_{\sigma_{1}}^{\Delta}\left(P_{\sigma} K_{\mathrm{Ai}}\right)^{n}
\end{aligned}
$$

where to go from the first two lines to the third we have used repeatedly (82) for each of the $n+1$ integrals.

$\S$ In the large $\Delta$ limit, the proper variable becomes $\bar{X}=\hat{X} \Delta^{-1 / 3}$. However we do not focus on this limit here. 


\section{The case of different endpoints $X \neq 0$}

We consider now the case where the height at time $t_{2}$ is measured at $x=X \neq 0$ while the height at $t_{1}$ is measured at $x=0$, as in (12). One defines the rescaled position variable $\S$

$$
\hat{X}=\frac{X}{2\left(t_{1} \Delta\right)^{2 / 3}}=\frac{X}{2\left(t_{2}-t_{1}\right)^{2 / 3}},
$$

and the definitions (14) still apply except that the definition of the scaled height difference variable $h$ becomes

$$
H_{2}-H_{1}=\left(\Delta t_{1}\right)^{1 / 3}\left(h-\hat{X}^{2}\right)=\frac{X^{2}}{4 \Delta t_{1}}+\left(\Delta t_{1}\right)^{1 / 3} h,
$$

where we recall $\Delta t_{1}=t_{2}-t_{1}$. We again define $\hat{\mathcal{P}}_{\Delta}\left(\sigma_{1}, \sigma_{2}\right)$ by Eq. (16). As in [1], the JPDF of $h_{1}$ and $h, P_{\Delta}\left(\sigma_{1}, \sigma\right)$, is still defined by (18), hence $\sigma$ is associated to $h$. The relation between $\sigma_{2}$ and $\sigma$ is thus changed and one has

$$
\mathcal{P}_{\Delta}\left(\sigma_{1}, \sigma\right)=\hat{\mathcal{P}}_{\Delta}\left(\sigma_{1}, \sigma_{2}=\frac{\sigma_{1}+\left(\sigma-\hat{X}^{2}\right) \Delta^{1 / 3}}{(1+\Delta)^{1 / 3}}\right),
$$

and (19) is still valid.

The Johansson formula (32) is still valid for $X \neq 0[3]$ but with generalized functions $\|$ which we denote by an index $\hat{X}$. One has

$$
S_{3}^{\hat{X}}(x, y)=e^{(y-x) \hat{X} \Delta^{-1 / 3}} K_{\mathrm{Ai}}\left(\sigma_{1}-x, \sigma_{1}-y\right) .
$$

We display the others in their similarity transformed form, defined as in (35), where we also changed $s \rightarrow \Delta^{1 / 3} s$ in the integrals

$$
\begin{gathered}
\tilde{S}_{1}^{\hat{X}}(x, y)=-\Delta^{1 / 3} \int_{0}^{\infty} d s e^{(s-x) \hat{X}} K_{\mathrm{Ai}}\left(\sigma_{1}-s \Delta^{1 / 3}, \sigma_{1}-x \Delta^{1 / 3}\right) K_{\mathrm{Ai}}(\sigma+s, \sigma+y), \\
\tilde{T}_{1}^{\hat{X}}(x, y)=\Delta^{1 / 3} \int_{-\infty}^{0} d s e^{(s-x) \hat{X}} K_{\mathrm{Ai}}\left(\sigma_{1}-s \Delta^{1 / 3}, \sigma_{1}-x \Delta^{1 / 3}\right) K_{\mathrm{Ai}}(\sigma+s, \sigma+y), \\
\tilde{S}_{2}^{\hat{X}}(x, y)=\tilde{S}_{2}(x, y)=K_{\mathrm{Ai}}(\sigma+x, \sigma+y) .
\end{gathered}
$$

Hence we see that, while $S_{2}$ is unchanged, in $\tilde{S}_{1}^{\hat{X}}$ and $\tilde{T}_{1}^{\hat{X}}$ the Airy kernel $K_{\mathrm{Ai}}\left(\sigma_{1}-s \Delta^{1 / 3}, \sigma_{1}-x \Delta^{1 / 3}\right)$ has been multiplied by $e^{\hat{X}(s-x)}$. By following the same steps as for $\hat{X}=0$, it is easy to show that the large positive $\sigma_{1}$ tail is given by its prediction in [1] as

$$
\hat{\mathcal{P}}\left(\sigma_{1}, \sigma_{2}\right)=\mathcal{P}_{\Delta, \hat{X}}^{(1)}\left(\sigma_{1}, \sigma\right)+O\left(e^{-\frac{8}{3} \sigma_{1}^{3 / 2}}\right)
$$

$\|$ we set there $\eta_{1}=0, \Delta \eta=\hat{X}$ and $\Delta \xi=\sigma-\hat{X}^{2}$ there, and use $\delta=\alpha \Delta \eta+0^{+}$. 
with

$$
\begin{aligned}
\mathcal{P}_{\Delta, \hat{X}}^{(1)}\left(\sigma_{1}, \sigma\right)=1+ & \left(F_{2}^{(1)}\left(\sigma_{2}^{\prime}\right)-1\right)-F_{2}(\sigma) \operatorname{Tr} P_{\sigma_{1}} K_{\mathrm{Ai}}+F_{2}(\sigma) \Delta^{1 / 3} \operatorname{Tr} P_{\sigma} K_{\sigma_{1}}^{\Delta, \hat{X}} \\
& +F_{2}(\sigma) \Delta^{1 / 3} \operatorname{Tr} P_{\sigma} K_{\mathrm{Ai}} P_{\sigma} K_{\sigma_{1}}^{\Delta, \hat{X}} P_{\sigma}\left(I-P_{\sigma} K_{\mathrm{Ai}} P_{\sigma}\right)^{-1}
\end{aligned}
$$

i.e. as in $(61)$ where the kernel $K_{\sigma_{1}}^{\Delta}$ is replaced by the $\hat{X}$-generalized kernel

$K_{\sigma_{1}}^{\Delta, \hat{X}}(u, v)=\int_{0}^{\infty} d y_{1} d y_{2} \operatorname{Ai}\left(-y_{1}+u\right) e^{\hat{X}\left(y_{2}-y_{1}\right)} K_{\mathrm{Ai}}\left(y_{1} \Delta^{1 / 3}+\sigma_{1}, y_{2} \Delta^{1 / 3}+\sigma_{1}\right) \operatorname{Ai}\left(-y_{2}+v\right)$,

exactly as defined in Eq. (140) in [1]. In Eq. (95)

$$
\sigma_{2}^{\prime}=\sigma_{2}+\frac{\Delta^{4 / 3}}{(1+\Delta)^{4 / 3}} \hat{X}^{2}=\frac{H_{2}}{2 t_{2}^{2 / 3}}+\frac{X^{2}}{4 t_{2}^{4 / 3}},
$$

as expected from the statistical tilt symmetry. Some details of this derivation are reported in Appendix B.

\section{Conclusion}

In this paper we showed that the tail of the two-time joint distribution of the height for the KPZ universality class obtained in $[1,2]$ is exact, as it agrees with the large $\sigma_{1}$ limit of the recently obtained rigorous two-time distribution [3]. This constitutes the first proof of the validity of the tail expression found in $[1,2]$ for the droplet initial condition, which up to now was only checked indirectly by comparison with experimental and numerical data in [2]. The approximations in [1,2] consisted in performing, within the RBA method, a partial (instead of full) summation over the eigenstates of the LL model (keeping only the ground state for the first time slice from $t=0$ to $t=t_{1}$ ). We conjectured in $[1,2]$, based on previous experience on the one-time observables, that this gives the exact tail for large $\sigma_{1}$. Thanks to the exact result found in [3], we showed here that this conjecture is indeed correct. The expansion at large $\sigma_{1}$ started in this paper opens the way to obtain a full solution, within the RBA method, of the multi-time problem, to be addressed in future publications.

\section{Acknowledgment}

We are very grateful to K. Johansson for very helpful discussions and exchanges.

This work is supported by LabEx ENS-ICFP:ANR-10-LABX-0010/ANR-10-IDEX0001-02 PSL* (J.D.N.) and ANR grant ANR-17-CE30-0027-01 RaMaTraF (P.L.D.) 


\section{Appendix A. How to recover the JPDF of $h_{1}$ and $h$ from the cumulative distribution}

The JPDF of $h_{1}$ and $h$ can be obtained from the cumulative distribution $\hat{\mathcal{P}}_{\Delta}$ as follows (where the limit $\lim _{t_{1}, t_{2} \rightarrow+\infty, \frac{t_{1}-t_{2}}{t_{1}}=\Delta}$ is implicit)

$$
\begin{aligned}
& P_{\Delta}\left(\sigma_{1}, \sigma\right)=\overline{\delta\left(h_{1}-\sigma_{1}\right) \delta(h-\sigma)}=\frac{1}{\left(1+\frac{1}{\Delta}\right)^{1 / 3}} \overline{\delta\left(h_{1}-\sigma_{1}\right) \delta\left(h_{2}-\frac{\sigma+\sigma_{1} \Delta^{-1 / 3}}{\left(1+\frac{1}{\Delta}\right)^{1 / 3}}\right)} \\
& =\frac{1}{\left(1+\frac{1}{\Delta}\right)^{1 / 3}} \partial_{\sigma_{1}} \partial_{\sigma_{2}} \hat{\mathcal{P}}_{\Delta}\left(\sigma_{1}, \sigma_{2}\right)=\frac{1}{\left(1+\frac{1}{\Delta}\right)^{1 / 3}} \partial_{\sigma_{1}} \partial_{\sigma_{2}} \mathcal{P}_{\Delta}\left(\sigma_{1}, \sigma=\sigma_{2}\left(1+\frac{1}{\Delta}\right)^{1 / 3}-\sigma_{1} \Delta^{-1 / 3}\right) \\
& =\left(\partial_{\sigma_{1}} \partial_{\sigma}-\Delta^{-1 / 3} \partial_{\sigma}^{2}\right) \mathcal{P}\left(\sigma_{1}, \sigma\right) .
\end{aligned}
$$

Note that $P_{\Delta}\left(\sigma_{1}, \sigma\right)=\frac{1}{\left(1+\frac{1}{\Delta}\right)^{1 / 3}} \hat{P}_{\Delta}\left(\sigma_{1}, \sigma_{2}\right)$, where $\hat{P}_{\Delta}$ is the JPDF of $h_{1}$ and $h_{2}$

$$
\hat{P}_{\Delta}\left(\sigma_{1}, \sigma_{2}\right)=\lim _{t_{1}, t_{2} \rightarrow+\infty, \frac{t_{1}-t_{2}}{t_{1}}=\Delta} \overline{\delta\left(h_{1}-\sigma_{1}\right) \delta\left(h_{2}-\sigma_{2}\right)} .
$$

Appendix B. More details for the case with non-zero endpoint $\hat{X} \neq 0$

The large $\sigma_{1}$ expansion proceeds exactly as for $X=0$. Since $\tilde{S}_{2}$ is unchanged, it thus leads, as in (60), to the expression

$$
\begin{aligned}
\mathcal{P}_{\Delta}\left(\sigma_{1}, \sigma\right) & =1+\operatorname{Tr}\left(\tilde{S}_{1}^{\hat{X}}-\tilde{T}_{1}^{\hat{X}}\right)+F_{2}(\sigma) \operatorname{Tr} P^{-} \tilde{T}_{1}^{\hat{X}}-F_{2}(\sigma) \operatorname{Tr} P^{-} S_{3}^{\hat{X}} \\
& +F_{2}(\sigma) \operatorname{Tr} P^{-} \tilde{T}_{1}^{\hat{X}} P^{+}\left(1-P^{+} \tilde{S}_{2} P^{+}\right)^{-1} P^{+} \tilde{S}_{2}+O\left(\epsilon^{2}\right) .
\end{aligned}
$$

Let us briefly examine each term. One has

$$
\begin{aligned}
& \operatorname{Tr}\left(\tilde{S}_{1}^{\hat{X}}-\tilde{T}_{1}^{\hat{X}}\right) \\
& =-\int_{z_{1}, z_{2}>0, s, x} e^{(s-x) \hat{X}} \operatorname{Ai}\left(\sigma_{1}-s \Delta^{1 / 3}+z_{1}\right) \operatorname{Ai}\left(\sigma_{1}-x \Delta^{1 / 3}+z_{1}\right) \operatorname{Ai}\left(\sigma+s+z_{2}\right) \operatorname{Ai}\left(\sigma+x+z_{2}\right) .
\end{aligned}
$$

We can now use the identity

$$
\begin{aligned}
& \int_{s} e^{s \hat{X}} \operatorname{Ai}\left(a-s \Delta^{1 / 3}\right) \operatorname{Ai}(b+s) \times \int_{x} e^{-x \hat{X}} \operatorname{Ai}\left(a-x \Delta^{1 / 3}\right) \operatorname{Ai}(b+x) \\
& =\frac{1}{(1+\Delta)^{2 / 3}}\left[\operatorname{Ai}\left(\frac{a+\Delta^{1 / 3} b}{(1+\Delta)^{1 / 3}}-\frac{\Delta^{1 / 3}}{(1+\Delta)^{4 / 3}} \hat{X}^{2}\right)\right]^{2}
\end{aligned}
$$

with $a=\sigma_{1}+z_{1}$ and $b=\sigma+z_{2}$. Proceeding as in Section 6.2 one obtains

$$
\operatorname{Tr}\left(\tilde{S}_{1}-\tilde{T}_{1}\right)=-\operatorname{Tr} P_{\sigma_{2}^{\prime}} K_{\mathrm{Ai}}=F_{2}^{(1)}\left(\sigma_{2}^{\prime}\right)-1 \quad, \quad \sigma_{2}^{\prime}=\sigma_{2}+\frac{\Delta^{4 / 3}}{(1+\Delta)^{4 / 3}} \hat{X}^{2}
$$

where we have used that $\sigma_{2}=\frac{\sigma_{1}+\left(\sigma-\hat{X}^{2}\right) \Delta^{1 / 3}}{(1+\Delta)^{1 / 3}}$.

Moreover we find, since the prefactor in (90) does not affect the trace of the kernel

$$
\operatorname{Tr} P^{-} S_{3}^{\hat{X}}=\operatorname{Tr} P_{\sigma_{1}} K_{\mathrm{Ai}}
$$


Next, from (92) one has

$$
\operatorname{Tr} P^{-} \tilde{T}_{1}^{\hat{X}}=\Delta^{1 / 3} \int_{x, s>0} e^{(x-s) \hat{X}} K_{\mathrm{Ai}}\left(\sigma_{1}+\Delta^{1 / 3} s, \sigma_{1}+\Delta^{1 / 3} x\right) K_{\mathrm{Ai}}(\sigma-s, \sigma-x) .
$$

On the other hand, from the definition (96), our result is

$$
\begin{aligned}
\Delta^{1 / 3} \operatorname{Tr} P_{\sigma} K_{\sigma_{1}}^{\Delta, \hat{X}} & =\Delta^{1 / 3} \int_{0}^{\infty} d v \int_{0}^{\infty} d y_{1} d y_{2} \operatorname{Ai}\left(-y_{1}+v+\sigma\right) \\
& \times e^{\hat{X}\left(y_{2}-y_{1}\right)} K_{\mathrm{Ai}}\left(y_{1} \Delta^{1 / 3}+\sigma_{1}, y_{2} \Delta^{1 / 3}+\sigma_{1}\right) \operatorname{Ai}\left(-y_{2}+v+\sigma\right) \\
& =\Delta^{1 / 3} \int_{0}^{\infty} d y_{1} d y_{2} e^{\hat{X}\left(y_{2}-y_{1}\right)} K_{\mathrm{Ai}}\left(y_{1} \Delta^{1 / 3}+\sigma_{1}, y_{2} \Delta^{1 / 3}+\sigma_{1}\right) \\
& \times K_{\mathrm{Ai}}\left(-y_{1}+\sigma,-y_{2}+\sigma\right)
\end{aligned}
$$

which is identical to (B.6).

Finally, as in Section 6.2, one can prove

$$
\operatorname{Tr} P^{+} \tilde{S}_{2} P^{-} \tilde{T}_{1}^{\hat{X}}\left(P^{+} \tilde{S}_{2}\right)^{n}=\Delta^{1 / 3} \operatorname{Tr} P_{\sigma} K_{\mathrm{Ai}} P_{\sigma} K_{\sigma_{1}}^{\Delta, \hat{X}}\left(P_{\sigma} K_{\mathrm{Ai}}\right)^{n} .
$$

It is easy to see, following the same steps, that (78) becomes

$$
\left(\tilde{S}_{2} P^{-} \tilde{T}_{1}^{\hat{X}}\right)(x, y)=\Delta^{1 / 3}\left\langle\mathrm{Ai}_{\sigma+x}\left|P^{+} K_{\sigma_{1}, \sigma}^{\Delta, \hat{X}} P^{+}\right| \mathrm{Ai}_{\sigma+y}\right\rangle .
$$

The same steps as in Section 6.2 then immediately lead to (B.8). Hence we obtain that (B.1) is identical to our result (95)-(96).

\section{References}

[1] J. De Nardis, P. Le Doussal, J. Stat. Mech. (2017) 053212, arXiv:1612.08695

[2] J. De Nardis, P. Le Doussal, K. A. Takeuchi, Phys. Rev. Lett. 118, 125701 (2017), arXiv:1611.04756

[3] K. Johansson, arXiv:1802.00729 (2018)

[4] M. Kardar, G. Parisi and Y.C. Zhang, Phys. Rev. Lett. 56889 (1986).

[5] M. Prahofer and H. Spohn, Phys. Rev. Lett. 844882 (2000), arXiv:0212519; M. Prahofer and H. Spohn, J. Stat. Phys. 1081071 (2002); M. Prahofer and H. Spohn, 115255 (2004).

[6] P. Calabrese, P. Le Doussal and A. Rosso, EPL 9020002 (2010), arXiv:1002.4560.

[7] V. Dotsenko, EPL 9020003 (2010), arXiv:1003.4899; V. Dotsenko and B. Klumov, J. Stat. Mech. P03022 (2010).

[8] T. Sasamoto and H. Spohn, Phys. Rev. Lett. 104230602 (2010), arXiv:1002.1883; T. Sasamoto and H. Spohn, Nucl. Phys. B 834523 (2010), arXiv:1002.1879 ; T. Sasamoto and H. Spohn, J. Stat. Phys. 140209 (2010), arXiv:1002.1873.

[9] G. Amir, I. Corwin, J. Quastel, Comm. Pure Appl. Math 64466 (2011), arXiv:1003.0443.

[10] P. Calabrese, M. Kormos and P. Le Doussal, EPL 10710011 (2014), arXiv:1405.2582.

[11] P. Calabrese and P. Le Doussal, Phys. Rev. Lett. 106250603 (2011), arXiv:1104.1993.

[12] P. Le Doussal and P. Calabrese, J. Stat. Mech. P06001(2012), arXiv:1204.2607.

[13] P. Calabrese and P. Le Doussal, J. Stat. Mech. P05004 (2014), arXiv:1402.1278.

[14] V. Dotsenko, J. Stat. Mech. P11014 (2012), arXiv:1209.3603.

[15] J. Ortmann, J. Quastel and D. Remenik, Ann. Appl. Probab. 26507 (2016), arXiv:1407.8484

[16] T. Imamura, T. Sasamoto, Phys. Rev. Lett. 108190603 (2012), arXiv:1111.4634; J. Stat. Phys. 150908 (2013), arXiv:1210.4278. 
[17] J. Baik and E.M. Rains, J. Stat. Phys. 100523 (2000), arXiv:math/0003130.

[18] A. Borodin, I. Corwin, P. L. Ferrari. B. Veto, arXiv:1407.6977.

[19] C.A. Tracy and H. Widom, Comm. Math. Phys. 159151 (1994), arXiv:hep-th/9211141.

[20] S. Prolhac and H. Spohn, J. Stat. Mech. P01031 (2011), arXiv:1011.401.

[21] S. Prolhac and H. Spohn, J. Stat. Mech. P03020 (2011), arXiv:1101.4622.

[22] V. Dotsenko, J. Phys. A. 46355001 (2013), arXiv:1304.6571.

[23] T. Imamura, T. Sasamoto, H. Spohn, J. Phys. A: Math. Theor. 46 355002, arXiv:1305.1217.

[24] I. Corwin, Rand. Mat.: Theo. Appl. 1, 1130001 (2012).

[25] K. Matetski, J. Quastel, D. Remenik, The KPZ fixed point, arXiv:1701.00018.

[26] K. A. Takeuchi and M. Sano, Phys. Rev. Lett. 104230601 (2010), arXiv:1001.5121 ; K. A. Takeuchi, M. Sano, T. Sasamoto, and H. Spohn, Sci. Rep. (Nature) 134 (2011).

[27] K. A. Takeuchi, M. Sano, J. Stat. Phys. 147853 (2012), arXiv:1203.2530.

[28] T. Halpin-Healy, K. A. Takeuchi, J. Stat. Phys. 160794 (2015), arXiv:1505.01910.

[29] E. H. Lieb and W. Liniger, Phys. Rev. 1301605 (1963).

[30] P. Calabrese and J.-S. Caux, Phys. Rev. Lett. 98150403 (2007), arXiv:cond-mat/0612192; P. Calabrese and J.-S. Caux, J. Stat. Mech. P08032 (2007), arXiv:0707.4115.

[31] K. A. Takeuchi, Phys. Rev. Lett. 110210604 (2013), arXiv:1301.5081.

[32] K. A. Takeuchi, T. Akimoto, J. Stat. Phys. 1641167 (2016), arXiv:1509.03082.

[33] K. Johansson, Commun. Math. Phys. 1432 (2016), arXiv:1502.00941.

[34] P. L. Ferrari, H. Spohn, SIGMA 12 (2016) 074, arXiv:1602.00486.

[35] V. Dotsenko, J. Stat. Mech. P06017 (2013), arXiv:1304.0626.

[36] V. Dotsenko, arXiv:1507.06135.

[37] V. Dotsenko, J.Phys. A: Math. Theor. 4927 (2016), arXiv:1603.08945.

[38] N. A. Slavnov, Theor. Math. Phys. 82273 (1990).

[39] L. Piroli, P. Calabrese. J. Phys. A: Math. Theor. 48, 454002 (2015). arXiv:1506.06539

[40] J. Baik, Z. Liu, Multi-point distribution of periodic TASEP, arXiv:1710.03284

[41] P. Le Doussal, Phys. Rev. E 96, 060101 (2017), arXiv:1709.06264.

[42] K. Johansson, private communication, has shown that [3] recovers the function $R_{1 / 3}$ defined in [1,2] in the double limit $\Delta \rightarrow+\infty$ and $\sigma_{1} \rightarrow+\infty$.

[43] The estimate $C_{\infty} \approx 0.6$ was quoted in [2] as being consistent with numerical data obtained there, with the theory of [2], and with previous experimental data (Takeuchi Private Comm. 2016).

[44] The estimate $C_{\infty}=0.626 \pm 0.003$ was obtained by Halpin-Healy (Private Comm. 2017) simulating the $1+1$ directed polymer subject to exponential site disorder. 